\title{
Isolation and genetic analysis of a variant porcine epidemic diarrhea virus in China
}

\author{
Ren-feng $\mathrm{Li}^{1}$, Xiang-qin $\mathrm{Tian}^{3}$, Yong Liu ${ }^{3}$, Jing $\mathrm{Xu}^{2}$, Dong-yang Liu ${ }^{1}$, \\ ${ }^{1}$ College of Veterinary Medicine, Henan Institute of Science and Technology, Xinxiang 453003, China \\ ${ }^{2}$ Medical Research Center, Xinxiang Medical College, Xinxiang 453003, China \\ ${ }^{3}$ Department of Respiration, Central Hospital of Zhoukou city, Zhoukou 466000, China
}

\begin{abstract}
Porcine epidemic diarrhea virus (PEDV) is having a severe effect on the pig breeding industry in central China. The mucosa and the content of the small intestine from newborn pre-weaned piglets with diarrhea were tested for the presence of PEDV by molecular and morphologic methods, and found to be positive. Negative-staining electron microscopy (EM) revealed the presence of coronavirus-like particles in the samples. The result of molecular detection by nested RT-PCR based on the amplification of the $\mathrm{M}$ gene was positive. Using a novel alternative method we successfully propagated the PEDV strain $(\mathrm{CH} / \mathrm{QX}-2)$ in Vero cells, confirmed by ultrathin sections of the cells and Immunofluorescence assay (IFA). Phylogenetic analysis based on the partial S gene showed that the $\mathrm{CH} / \mathrm{QX}-2$ isolate was genetically closer to strains more commonly found in China, but differed genetically from two domestic strains (CH/S, 1986 and LZC, 2007), Korean strains (DR13, 2007), and the vaccine strain (CV777 vs) currently being used in China. $\mathrm{CH} / \mathrm{QX}-2$ formed a unique clade in the derived phylogenetic tree indicating that the $\mathrm{CH} / \mathrm{QX}-2$ strain currently circulating in central China is a new variant of PEDV. This study extends current knowledge on the diversity and epidemiology of PEDV.
\end{abstract}

Key words: isolation, genetic, variant, porcine epidemic diarrhea virus

\section{Introduction}

As a member of the family Coronaviridae, porcine epidemic diarrhea virus (PEDV) can induce an acute and highly contagious enteric disease of swine with a high mortality in piglets, which is characterized by severe enteritis, vomiting, and watery diarrhea (Ducatelle et al. 1981). The disease was first recognized in
England in 1971 (Pensaert et al. 1978), and since then, outbreaks have been reported in Europe and Asia (Bridgen et al. 1993, Puranaveja et al. 2009, Chen et al. 2010), and more recently in the United States (Wang et al. 2014). In China, although a periodic vaccination strategy has been applied nationwide to control the disease in pig farms, PEDV outbreaks have continued to occur causing large financial losses in the

Correspondence to: R.F. Li, e-mail: lirenfeng@sina.com 
Table 1. The primers used in this study.

\begin{tabular}{llc}
\hline \multicolumn{1}{c}{ Primer names } & \multicolumn{1}{c}{ Nucleotide sequence (5'-3') } & Size of product (bp) \\
\hline PEDV-Out-F & \multicolumn{1}{c}{853} \\
PEDV-Out-R ${ }^{\mathrm{a}}$ & ACACCTATAGGGCGCCTGTA & 412 \\
\hline PEDV-in-F & AACCCTAAGAGGGGCATAGA & \\
PEDV-in- $\mathrm{R}^{\mathrm{b}}$ & GGGCGCCTGTATAGAGTTTA & 894 \\
\hline PEDV-sF & AGACCACCAAGAATGTGTCC & \\
PEDV-sR & TTCTGAGTCATGAACAGCCAAT & \\
\hline
\end{tabular}

${ }^{\mathrm{a}}$ outer primer pairs used for nested-RT-PCR; ${ }^{\mathrm{b}}$ inner primer pairs used for nested-RT-PCR

Chinese swine industry. Since 2010, PEDV infection has become one of the most significant diseases affecting pig farming in central China (Sun et al. 2012). The PEDV genome contains at least seven open reading frames (ORFs), encoding four structural proteins [spike (S), envelope (E), membrane (M), and nucleocapsid $(\mathrm{N})$ ], and three non-structural proteins (replicases 1a and 1b and ORF3) (Song et al. 2012). The spike protein $(\mathrm{S})$ is the major structural protein of PEDV and consists of 1383 amino acids. Similar to other coronaviruses, the S protein of PEDV can be divided into three domains, including a large outer domain, a transmembrane domain and a short cytoplasm domain at the carboxy terminus. It has been demonstrated to have four neutralizing epitopes on the surface of $S$ protein (aa 499-638, 748-755, 764-771, and 1,368-1,374) (Sun et al. 2008), which are pivotal in receptor binding and cell entry, host-cell fusion, as well as the induction of neutralizing antibodies (Godet et al. 1994, Chang et al. 2002, Sun et al. 2007). Therefore, the $\mathrm{S}$ protein is a suitable candidate for examining the epidemiological status in the field, strain diversity and the association between gene mutations and viral antigenicity of PEDV (Park et al. 2007).

Molecular techniques have been commonly used diagnostically for identifying the presence of the PEDV genome. However, morphological methods are needed to confirm the identity of the pathogen and to further investigate pathogenesis. Combining molecular detection and morphologic methods, the present study describes the isolation of a PEDV strain currently circulating in the central region of China by optimizing the isolation procedures. Based on a partial $\mathrm{S}$ gene sequence, phylogenetic analysis has indicated that the isolated PEDV strain may be a new variant. The amino acid mutations in the neutralizing epitope regions may be the basis of its altered antigenicity and consequent resistance to vaccination. These findings may be useful for understanding its epidemiology as well as for the design of new and more effective vaccines.

\section{Materials and Methods}

\section{Specimen source}

An outbreak was observed in a swine farm from the central region of China, and characterized by acute watery diarrhea and high mortality rates among neonatal piglets. The specimens (small intestine and its contents) were sent to the Key Laboratory of Animal Immunology of the Ministry of Agriculture (Henan Academy of Agricultural Sciences, Zhengzhou, China) for the detection of PEDV.

\section{Morphologic observation of virus by TEM}

Samples were prepared for electron microscopy using negative staining, following a previously described procedure with slight modifications (Derbyshire 1988). The scraped-off mucosa and the content from the small intestine were pooled, diluted 1:5 in phosphate-buffered saline (PBS; 0.1 M, pH 7.2) to make $10 \%(\mathrm{~V} / \mathrm{V})$ suspensions. The suspensions were vortexed and after freezing and thawing three times clarified by centrifugation at $10,000 \times \mathrm{g}$ for $5 \mathrm{~min}$. Clarified supernatant fluids were placed on parlodion-coated grids in $50 \mu \mathrm{l}$ aliquots. After adsorption for $5 \mathrm{~min}$, the grids were negatively stained with $2 \%$ phosphotungstic acid ( $\mathrm{pH} 6.6$ ) for 2 min and subsequently viewed with an H-7500 transmission electron microscope (TEM).

\section{Nested RT-PCR for the detection of PEDV in the samples}

The mucosal scrapings and the small intestine contents were pooled, diluted 1:5 in phosphate-buffered saline (PBS; 0.1 M, pH 7.2), and homogenized by ultrasonication. The suspensions were vortexed and after freezing and thawing three times clarified by centrifugation for $10 \mathrm{~min}$ at $4,800 \times \mathrm{g}$. PEDV RNA was 
Table 2. PEDV strains used in this study for sequence alignment and phylogenetic analysis.

\begin{tabular}{cccccc}
\hline Reference Strains & Accession No & Origin & Reference Strains & Accession No & Origin \\
\hline ZJCZ4 & JX524137.1 & China, 2011 & GD-1 & JX647847.1 & China, 2011 \\
LC & JX489155.1 & China, 2012 & AH2012 & KC210145.1 & China, 2012 \\
CHGD-01 & JX261936.1 & China, 2012 & USA/Colorado/2013 & KF272920.1 & USA, 2013 \\
AJ1102 & JX188454.1 & China, 2012 & CH/ZMDZY/11 & KC196276.1 & China, 2011 \\
CH/FJZZ-9/2012 & KC140102.1 & China, 2013 & BJ-2011-1 & JN825712.1 & China, 2011 \\
GD-A & JX112709.1 & China, 2012 & CV777 & AF353511.1 & Belgium, 2001 \\
DR13 & JQ023161.1 & Korea, 2008 & LZC & EF185992.1 & China, 2007 \\
JS-HZ2012 & KC210147.1 & China, 2012 & CH/FJND-3/2011 & JQ282909.1 & China, 2011 \\
GD-B & JX088695.1 & China, 2012 & CH/S & JN547228.1 & China, 1986 \\
\hline
\end{tabular}

extracted using TRIzol reagent in accordance with the manufacturer's instructions. Two pairs of oligonucleotide primers were designed for the amplification of membrane protein (M) gene of PEDV CV777 strain (GenBank No. AF353511) and were used for the nested RT-PCR in two amplification steps. The nucleotide sequences of the primers and the expected size of the amplified DNA fragments were shown in Table 1.

The PCR mixture contained $2.5 \mu \mathrm{l}$ of $10 \times \mathrm{Taq}$ DNA polymerase buffer, $3 \mathrm{mM}$ of $\mathrm{MgCl}_{2}, 2.0 \mu$ of dNTPs $(2.5 \mathrm{mM} / \mathrm{L}), 0.5 \mu \mathrm{l}$ of each specific primer $(10$ pmol), $1 \mu \mathrm{l}$ of Taq DNA polymerase, $2 \mu \mathrm{l}$ cDNA and brought to $25 \mu \mathrm{l}$ with autoclaved, filtered $(0.2 \mu \mathrm{m})$ distilled water. The amplification step was carried out using the following thermal cycles: the denaturation at 94 for 4 min was followed by 30 cycles of the amplification step (denaturation at 94 for $1 \mathrm{~min}$, annealing at 50 for $40 \mathrm{~s}$, and extension at 72 for $1 \mathrm{~min}$ ), a final extension step at 72 for $5 \mathrm{~min}$. The product of the first reaction $(2 \mu \mathrm{l})$ was used as a template for the second amplification; the following thermal cycles were carried out: the denaturation step at 94 for 4 min followed by 30 cycles of the amplification step (denaturation at 94 for $30 \mathrm{~s}$, annealing at 55 for $30 \mathrm{~s}$, and extension at 72 for 40 s), a final extension step at 72 for $5 \mathrm{~min}$. The size of the amplified products in the first and the second reaction was $853 \mathrm{bp}$ and $412 \mathrm{bp}$, respectively.

\section{Virus isolation}

Propagation of PEDV was attempted in Vero cells (Vero-E6) as previously described with modifications (Hofmann et al. 1988). Vero cells were cultured and maintained in Minimum Essential Medium (MEM) supplemented with $10 \%$ fetal bovine serum, $2 \mathrm{mM}$ L-Glutamine, $0.05 \mathrm{mg} / \mathrm{ml}$ Gentamicin, 10 unit $/ \mathrm{ml}$ penicillin, $10 \mathrm{~g} / \mathrm{ml}$ Streptomycin, and $0.25 \mathrm{~g} / \mathrm{ml} \mathrm{Am-}$ photericin. First, the monolayers were washed twice with PBS and then inoculated with virus suspension pre-treated with trypsin [10 $\mu \mathrm{g}$ of trypsin (1:250) was added to $1 \mathrm{ml}$ of diluted and filtered virus suspension at 37 for $30 \mathrm{~min}]$. After adsorption at 37 for $2 \mathrm{~h}$ at room temperature, maintenance medium [MEM; supplemented with $0.3 \%$ tryptose phosphate broth and $0.02 \%$ yeast extract, and containing $2.5 \mu \mathrm{g}$ of trypsin (1:250) per $\mathrm{ml}$ ] was added without removing the viral inoculum, and the cultures further incubated at 37. Control cultures were mock inoculated with the same volume of maintenance medium instead of viral inoculum and further treated in the same manner as virus-inoculated cultures.

\section{Ultrathin section and IFA}

Vero cells infected with the CH/QX-2 isolate were trypsinized at $24 \mathrm{~h}$ post infection and centrifuged for $5 \mathrm{~min}$ at $800 \times \mathrm{g}$. The cell pellets were resuspended in $0.01 \mathrm{M}$ PBS ( $\mathrm{pH} 7.2-7.4$ ) and centrifuged for $5 \mathrm{~min}$ at $800 \times \mathrm{g}$. The resulting pellets were fixed in $2.5 \%$ glutaraldehyde in $0.1 \mathrm{M} \mathrm{Na}$ cacodylate buffer ( $\mathrm{pH} 7.4)$ and post-fixed in $1 \%$ osmium tetroxide for $90 \mathrm{~min}$. The samples were dehydrated in an ascending ethanol series, followed by propylene oxide and embedded in Eponate $12^{\mathrm{TM}}$. Ultrathin sections were stained with uranyl acetate and lead citrate, and examined by TEM (H- 7500).

IFA was used to detect PEDV in the fixed-methanol infected Vero cells utilizing a 1:1000 dilution of mouse anti-S monoclonal antibody specific for PEDV (Cat No: 9191, MEDIAN Diagnostics Inc, Korea) and a 1:100 dilution of fluorescein isothiocyanate-conjugated goat anti-mouse IgG (Cat no: F0257, Sigma). Vero cells without virus were similarly treated and used as an antigen negative control. The results were obtained using a fluorescence microscope.

\section{Cloning and sequence analysis of the partial $S$ gene for PEDV}

Viral RNA extraction was performed with $50 \mu \mathrm{l}$ of virus isolates propagated in the Vero cell cultures 


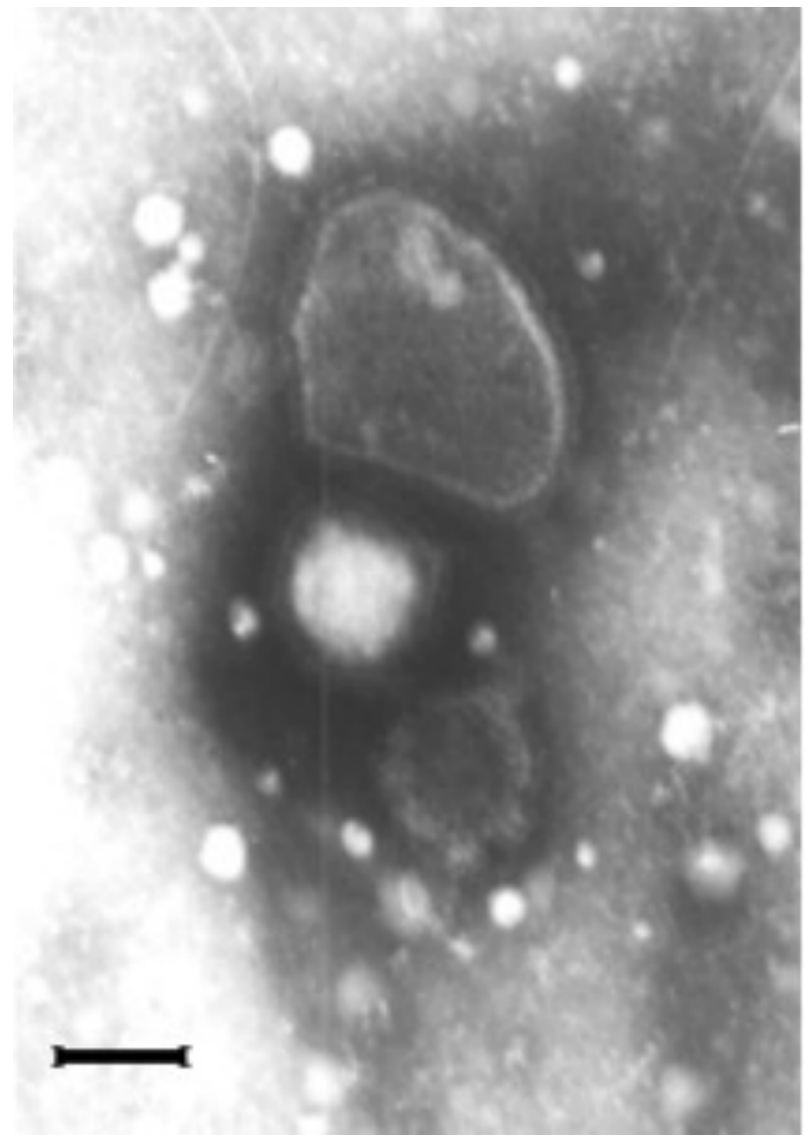

Fig. 1. Negatively stained virus particles in the small intestine homogenates from ill piglet with diarrhea. Some virus particles with crown-shaped spikes were viewed by TEM. $($ Bar $=100 \mathrm{~nm})$.

using the TRIzol reagent following the manufacture's instruction. Synthesis of the first-strand cDNA of the partial S gene was carried out by reverse transcription using TAKARA Reverse Transcription Kit (Code No. RR014A). The primers for amplifying partial S gene were designed based on the genome of PEDV CV777 (GenBank No. AF353511), and the size of the final fragment containing the three neutralizing epitopes of PEDV was 894 bp. Two sets of primers were synthesized by Sangon Biotech, China. The RT-PCR primers were given in Table 1 . The partial $\mathrm{S}$ gene was amplified under the following conditions: the denaturation at 95 for 5 min was followed by 30 cycles at 95 for $1 \mathrm{~min}, 55$ for $1 \mathrm{~min}, 72$ for $1 \mathrm{~min}$, and a final extension at 72 for $10 \mathrm{~min}$. The positive clones were sent to Sangon Biotech Company, China, for sequencing. All sequencing reactions were performed in duplicate.

Multiple-sequencing alignments were generated with Clustal W method by Meg-align 4.0 program in
DNASTAR. Phylogenetic tree was constructed by Molecular Evolutionary Genetics Analysis (MEGA) software (version 5.2.1) with the neighbor-joining method based on nucleotide sequences of the partial S gene (Tamura et al. 2011). To assess the relative support for each clade, bootstrap values were calculated from 1,000 replicate analyses. All strains used for phylogenetic analysis were presented. B-cell epitope prediction was performed by BepiPred 1.0 Server (http://www.cbs.dtu.dk/services/BepiPred/) (Larsen et al. 2006).

\section{Results}

\section{Morphologic characterization of virus}

TEM of the negatively stained preparations from the small intestinal contents revealed the presence of virus particles of approximately $100-140 \mathrm{~nm}$ in diameter carrying a number of ca $20 \mathrm{~nm}$ surface projections, in accord with the morphological characteristics of Coronaviridae members (Fig. 1).

\section{Molecular detection by nested-RT-PCR}

First 853-bp product was amplified using the outer primer pairs PEDV-out-F and PEDV-out-R. The second amplification, using nested or internal primer pairs PEDV-in-F and PEDV-in-R, produced a 412-bp PCR product. These were consistent with the expected fragment in length. Control reaction failed to produce a detectable amplification product (Fig. 2).

\section{Virus isolation and characterization}

Virus isolation was attempted on 40 PEDV-PCR positive intestine homogenates on Vero cells. One PEDV isolate designated as $\mathrm{CH} / \mathrm{QX}-2$ obtained from the small intestines of a 3-old day sucking piglets was successfully serially propagated in the Vero cell cultures.

Ultrathin sections of Vero cells after the ninth serial passage were examined by TEM. The cytoplasm of Vero cells infected with the CH/QX-2 isolate contained masses of virus particles with distinctive crown-shaped projections. Some virus particles were also seen at the surface of the cytoplasm membrane. No virions were observed in the Vero cells without CH/QX-2 infection (Fig. 3a). The Vero cells infected by $\mathrm{CH} / \mathrm{QX}-2$ also tested positive by IFA, whereas no fluorescence was observed in the control cultures (Fig. 3b). 
(a)

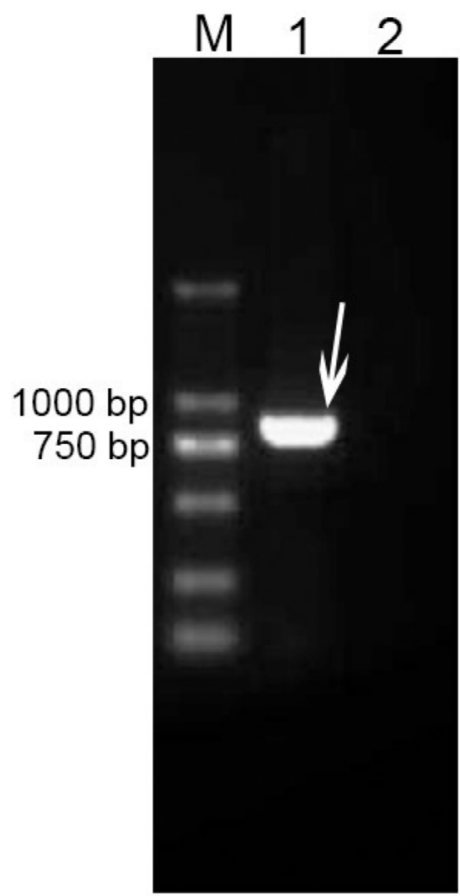

(b)

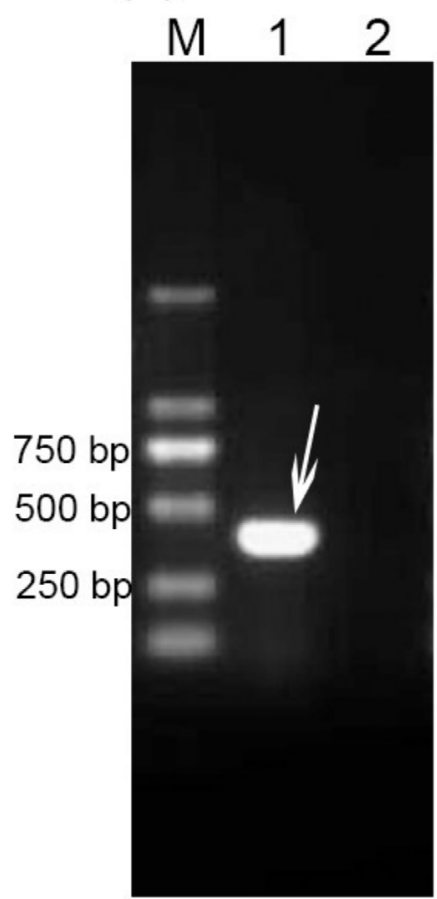

Fig. 2. Identification of PEDV in the content from small intestine of piglet with diarrhea by nested RT-PCR. (a) A specific fragment of about $853 \mathrm{bp}$ length was amplified using outer primer pairs of PEDV-Out-F: and PEDV-Out-R, which was indicated by white arrow. (b) A specific fragment of about $412 \mathrm{bp}$ length was amplified using inner primer pairs of PEDV-in-F, and PEDV-in-F, which was indicated by white arrows. M, DNA Marker DL 2000; lane 1, PEDV positive; Lane 2, Negative control (double distilled water).
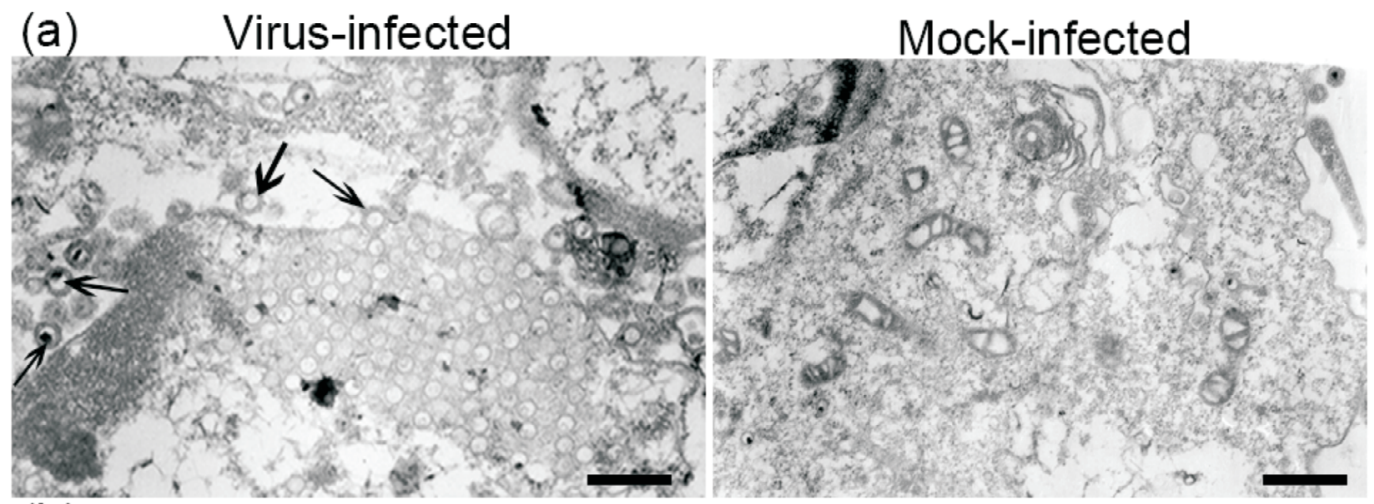

(b) Virus-infected Mock-infected
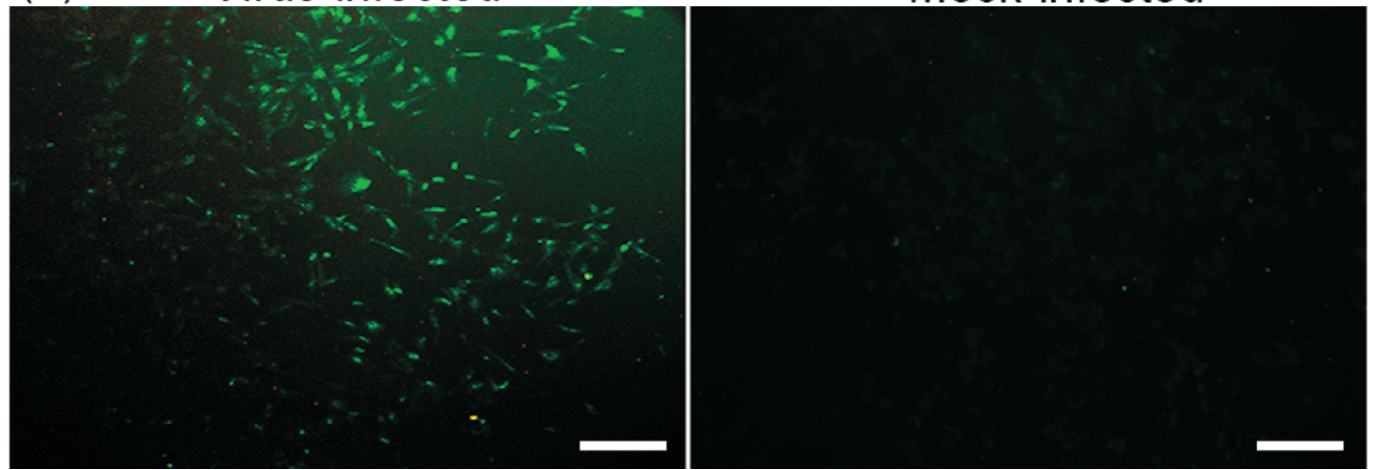

Fig. 3. The PEDV propagation successfully in the Vero cell cultures was confirmed by cell ultrathin section and IFA. (a) Multiply virus particles in the cytoplasm of Vero cells infected by $\mathrm{CH} / \mathrm{QX}-2$ were observed by TEM (left). No virions were viewed in the mock-infected cells (right). (Bar=333 nm). (b) Specific green fluorescence was visible in Vero cells $24 \mathrm{~h}$ post-infection with $\mathrm{CH} / \mathrm{QX}-2$ isolate (left), but no fluorescence was detected in control cultures (right). (Bar=100 $\mu \mathrm{m})$. 


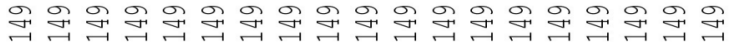

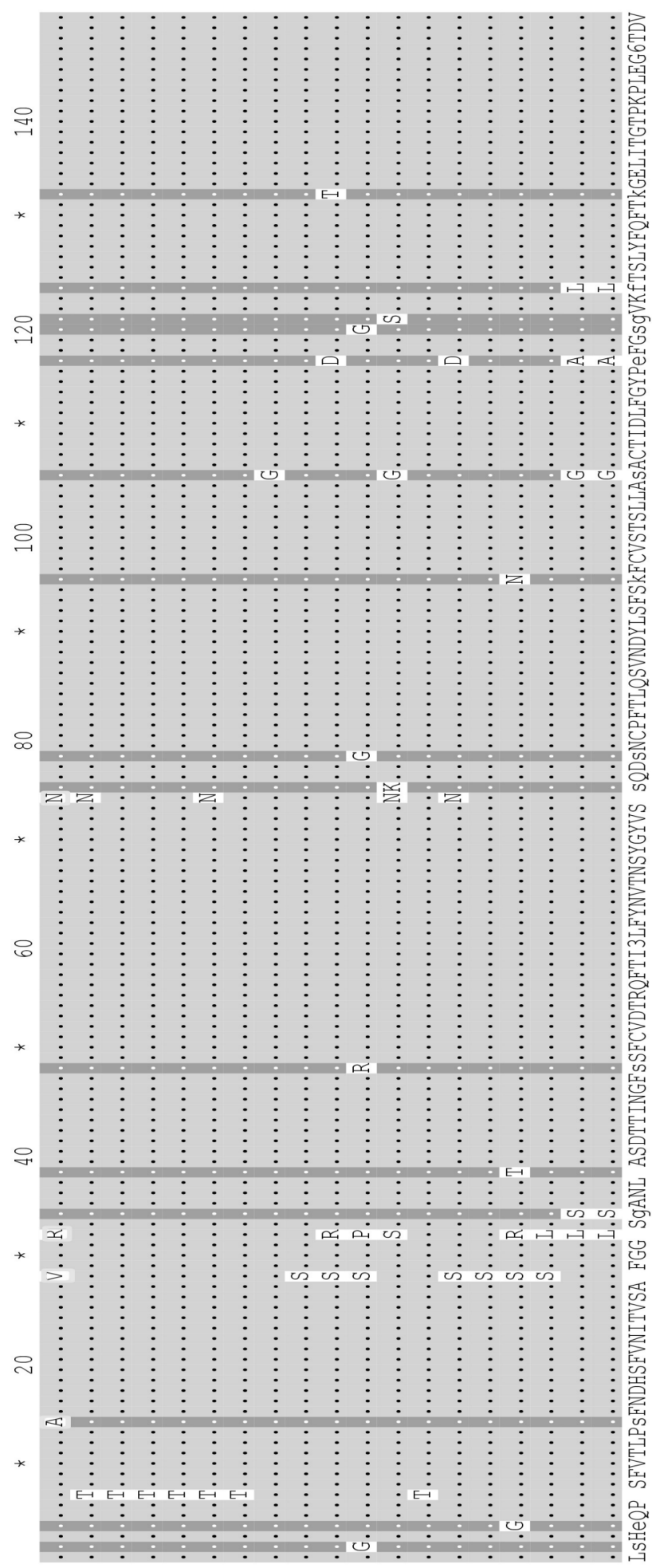

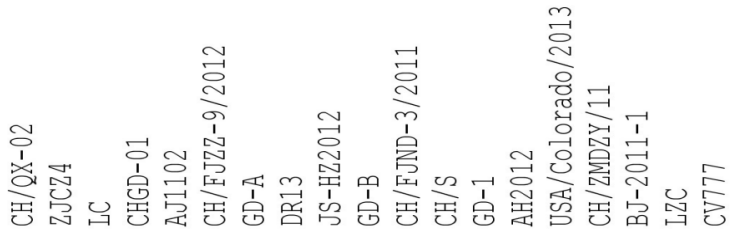

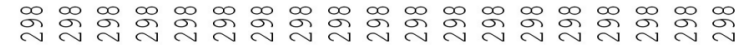
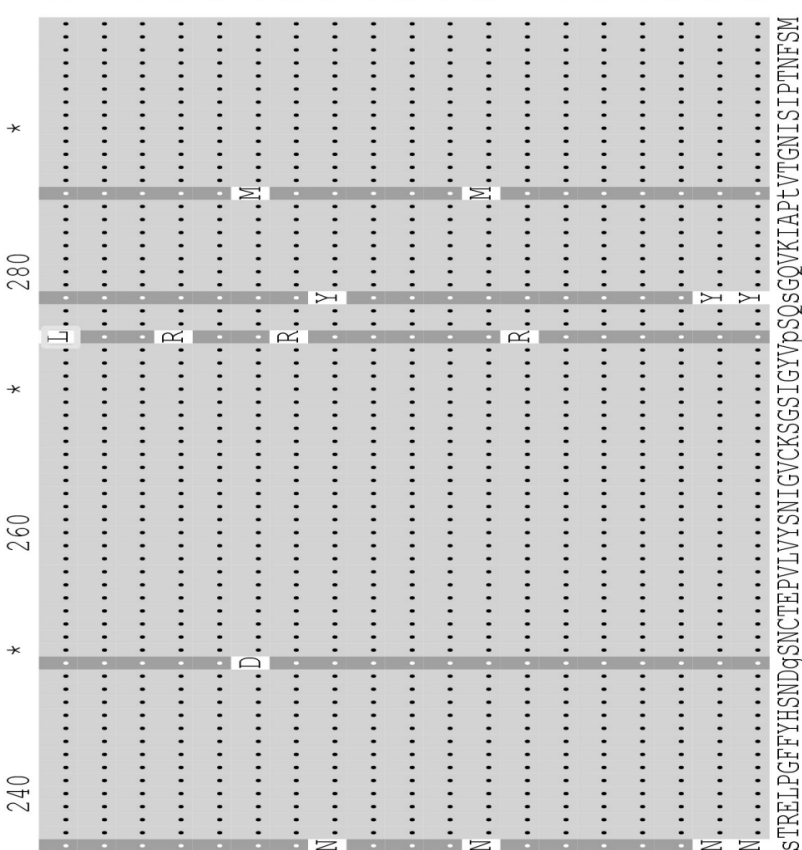

* $\quad \vdots \vdots$

๙্ণ
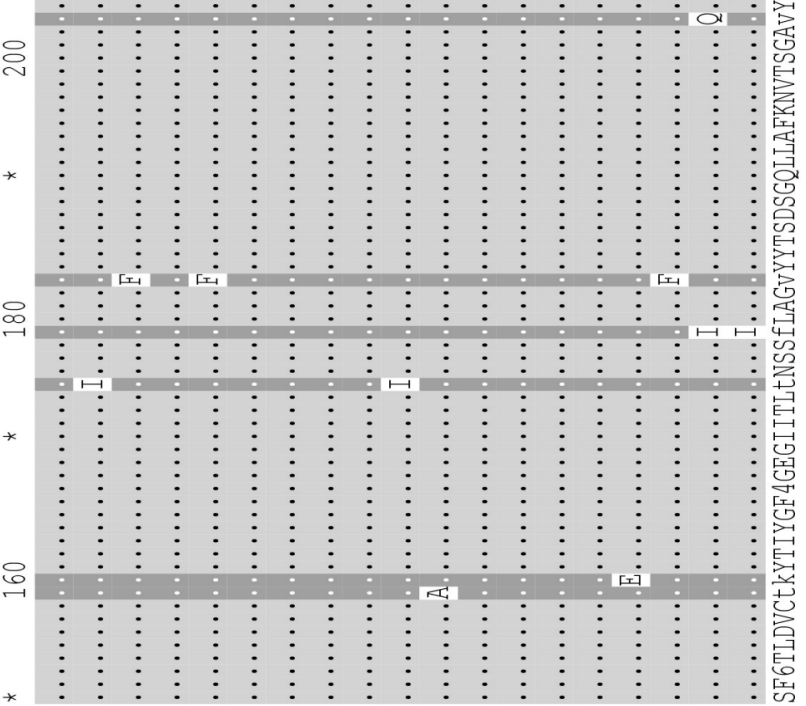

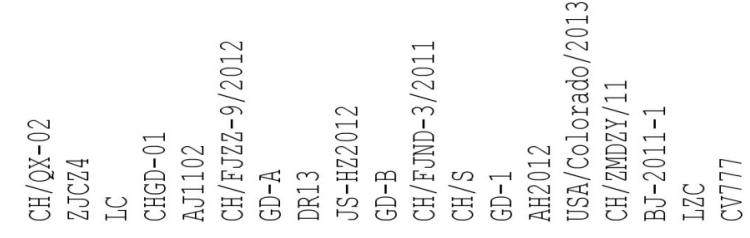

Fig. 4. Alignment of the partial S amino acid sequences of CH/QX-2 and PEDV reference strains by using Clustal W. The positions of the amino acid mutations in the partial $\mathrm{S}$ protein of $\mathrm{CH} / \mathrm{QX}-2$ isolate are marked by yellow square frame. 


\section{Cloning and phylogenetic analysis of the partial $S$ gene for PEDV}

The partial S gene amplified from the $\mathrm{CH} / \mathrm{QX}-2$ isolate gave an $894 \mathrm{nt}$ fragment. The sequence of the partial S gene, encoding a 298 amino acid polypeptide, was deposited in GenBank under the accession numbers KF484735, and the polypeptide contained three neutralizing epitopes (7-146, 245-252, 271-278), with $94.7 \%$ nucleotide and $94.3 \%$ deduced amino acid sequence similarity to the reference strains. Furthermore, the deduced amino acid sequences analysis showed that there was no amino acid change in the epitope aa 245-252 on the S protein of $\mathrm{CH} / \mathrm{QX}-2$. However, compared with the vaccine strain CV777, some variants were observed in the epitope regions aa 7-146 and 271-278, including $\mathrm{S} \rightarrow \mathrm{A}$ at $14, \mathrm{~A} \rightarrow \mathrm{V}$ at 28, $\mathrm{H} \rightarrow \mathrm{R}$ at $32, \mathrm{~K} \rightarrow \mathrm{N}$ at $74, \mathrm{P} \rightarrow \mathrm{L}$ at 274 . The predicted B-cell epitope of partial $\mathrm{S}$ protein at aa 7-146 was based on the amino acid sequence of CV777 by BepiPred 1.0b Server. The results showed that there were three B-cell epitopes with high scores which located at positions aa 22-30, 32-40, 64-75, respectively. The amino acid mutations at positions aa 28,32 , and 74 of the $\mathrm{S}$ protein of $\mathrm{CH} / \mathrm{QX}-2$ were distributed within the epitope regions predicted above, respectively (Fig. 4), which indicated that these changes might result in antigenicity difference between $\mathrm{CH} / \mathrm{QX}-2$ and the vaccine strain CV777.

A phylogenetic analysis based on the partial $\mathrm{S}$ amino acid sequence of $\mathrm{CH} / \mathrm{QX}-2$ and the reference strains gave two clusters. $\mathrm{CH} / \mathrm{QX}-2$ was distributed into G1, together with most of the other Chinese strains (GD-1, GD-A, CHGD-01, LC, AJ1102, ZJCZ4，CH/FJZZ-9/2012，JS-HZ2012，BJ-2011-1, GD-B, CH/ZMDZY/11, CH/FJND-3/2011, and AH2012), and one American strain (USA/Colorado/2013). Noticeably CH/QX-2 differed from two earlier domestic strains (CH/S, 1986 and LZC, 2007), the Korean strains (DR13, 2007), and the current vaccine strain (CV777 vs). Interestingly, CH/QX-2 formed an ndividual unique clade in phylogenetic tree (Fig. 5).

\section{Discussion}

PEDV has been detected frequently in many provinces in China and has become one of the most economically important viral causes of diarrhea in piglets (Gao et al. 2013). At this time the losses caused by the continuing rate of PEDV infection is a serious problem for many pig farms in China. In this study, the samples were collected from a pig breeding farm in Henan province, China, where a severe acute diarrhea outbreak associated with high morbidity
$(80-100 \%)$ and mortality $(60-100 \%)$ was observed in suckling piglets. For the whole-herd in this farm, a total number of 216 PEDV positive swine cases have been diagnosed in the following age groups: 93 suckling, 64 nursery, 36 grower/finisher, 23 sow/boar. Cleanliness, sanitation and disinfection measures were taken immediately following whole-herd exposure in preparation for the recovery phase post-infection. These actions reduce environmental load of virus and play a significant part in the restriction of viral transmission and biosecurity.

At present, RT-PCR based on the amplification of ORF3, M, S, and $\mathrm{N}$ gene has been used for the clinical diagnosis of PEDV (Lee et al. 2003, Park et al. 2008, Ogawa et al. 2009). However, morphological methods are still useful for the identification of an unknown virus. We initially examined the ultrastructure of lesions in the small intestine after an initial infection by PEDV. There was a loss of cell organelles and the terminal web. The tight junctions were lost, and microvilli had become fragmented or partially disappeared (date not shown).

It is necessary to diagnose rapidly and precisely in order to take steps to prevent the spread of PEDV. Due to the similarities in causative agents of diarrhea, the diagnosis of PED can not only depend on the clinical signs and histopathological lesions (Kweon et al. 1997). In this study nested RT-PCR was used for the detection of the PEDV based on a partial amplification of the $\mathrm{M}$ gene. We have applied this nested RT-PCR-based detection to clinical sample for diagnostic detection of PEDV and have found the positive rate of PEDV in all examined samples from 343 cases of diarrhea up to 78.6.

Trypsin is essential for the in vitro propagation of PEDV. The concentration of trypsin required in the culture medium is determined by the type of cell line and virus strain (Hofmann et al. 1988). In this study we found that treatment of the samples with $10 \mu \mathrm{g} / \mathrm{ml}$ of trypsin at 37 for 30 minutes before adding to the Vero cells was adequate to achieve an infection. Cytopathic effects (CPE) including cell fusion was subsequently observed in the infected Vero cells beyond the seventh passage (date not shown). Electron microscopy of infected cells at the ninth passage showed masses of virus particles distributed in a grid-like pattern in the cytoplasm (Fig. 3a), and the cells were positive by immunofluorescence (Fig. 3b). A sequence comparison with other PEDV strains selected from GenBank indicated that the partial S protein of $\mathrm{CH} / \mathrm{QX}-2$ shared highly homology with most Chinese strains and had a distant phylogenetic relationship with earlier Chinese strains $(\mathrm{CH} / \mathrm{S}$ and $\mathrm{LZC})$, the Korean strain (DR13), and the vaccine strain CV777. It is worth noting that the amino acid muta- 


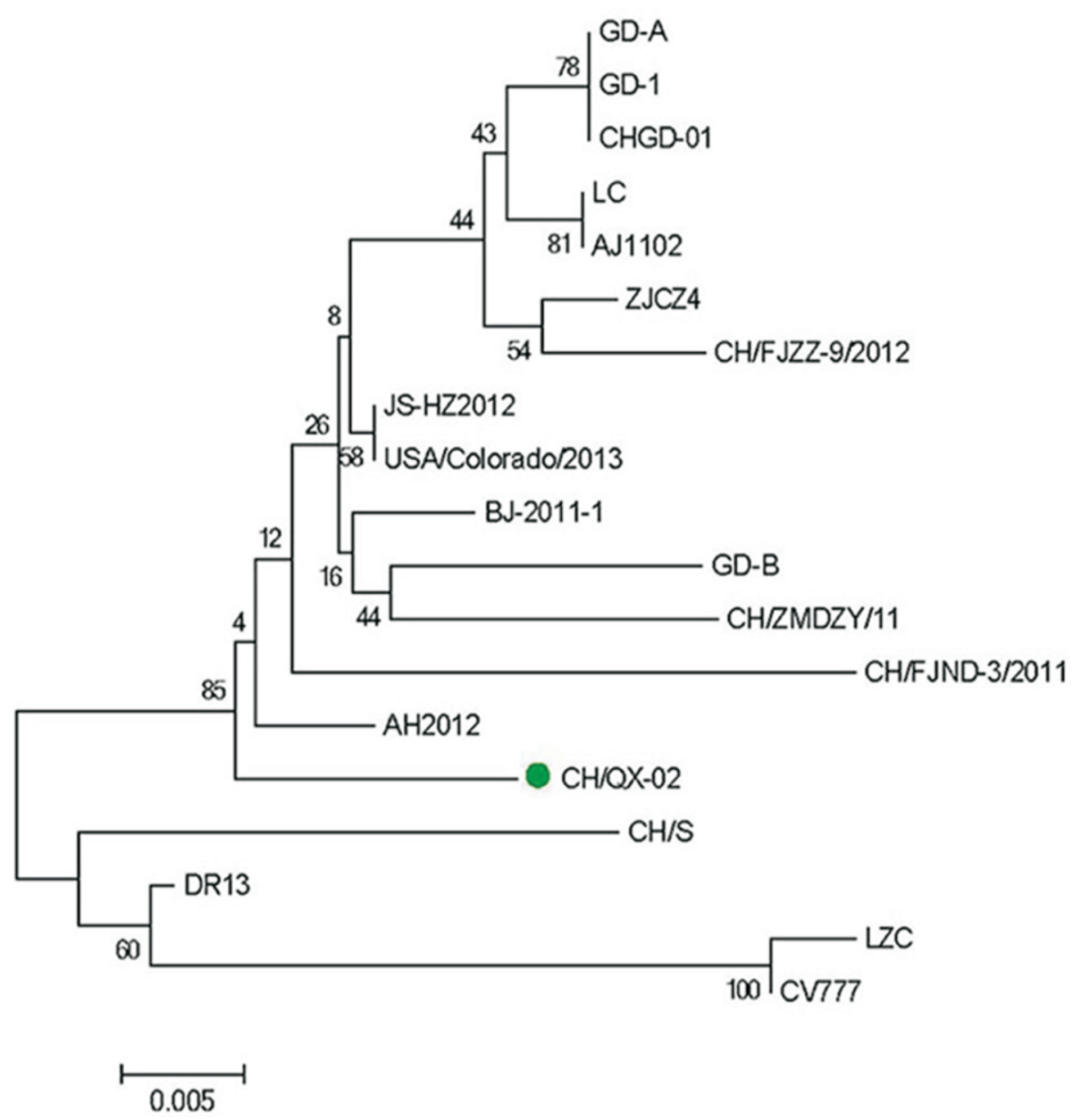

Fig. 5. Phylogenetic tree constructed based on the deduced amino acid sequences of the partial S genes from $\mathrm{CH} / \mathrm{QX}-2$ isolate and the referenced strains. The tree was generated by the neighbor-joining method with bootstrap analysis (1,000 replicates) and p-distance model. The $\mathrm{CH} / \mathrm{QX}-2$ isolate is highlighted with green triangle.

tions occurred within the B cell epitope regions and these could result in a change in the antigenicity of the new PEDV. Notably $\mathrm{CH} / \mathrm{QX}-2$ formed a unique clade in the phylogenetic tree which was closely related to AH2012 but did not belong to any subgroup (Fig. 5). Together, these findings indicate that $\mathrm{CH} / \mathrm{QX}-2$ is likely to be a new variant and most importantly may have a different antigencity to other strains and in particular to the strain used in the production of the current vaccine and hence has capacity to escape from immune surveillance and enhanced virulence which in turn causes immune response failure.

Using a novel alternative method we have successfully adapted PEDV to Vero cells allowing its proliferation by serial passage. This was confirmed by electron and immunofluorescence microscopy. Additionally, it was shown that although $\mathrm{CH} / \mathrm{QX}-2$ was genetically closer to most Chinese strains it differed from the earlier Chinese strains particularly the strain CV777 used for vaccine production. These findings indicate that the $\mathrm{CH} / \mathrm{QX}-2$ is a new variant of PEDV. There is an urgent need to study further the biologic characteristics, antigenicity, and mechanism of virulence variation of this new PEDV.

\section{Acknowledgments}

The authors would like to thank Dr Gregson, UCL-ION, London University, for revising the paper and advice in the work. This work was supported by grants from China Agriculture Research System (No. CARS-36), and the Pig Industry Technology System Innovation Team Project of Henan Province (S2012-06).

\section{References}

Bridgen A, Duarte M, Tobler K, Laude H, Ackermann M (1993) Sequence determination of the nucleocapsid protein gene of the porcine epidemic diarrhoea virus con- 
firms that this virus is a coronavirus related to human coronavirus $229 \mathrm{E}$ and porcine transmissible gastroenteritis virus. J Gen Virol 74: 1795-1804.

Chang SH, Bae JL, Kang TJ, Kim J, Chung GH, Lim CW, Laude H, Yang MS, Jang YS (2002) Identification of the epitope region capable of inducing neutralizing antibodies against the porcine epidemic diarrhea virus. Mol Cells 14: 295-299.

Chen J, Wang C, Shi H, Qiu H, Liu S, Chen X, Zhang Z, Feng L (2010) Molecular epidemiology of porcine epidemic diarrhea virus in China. Arch Virol 155: 1471-1476.

Derbyshire JB (1988) Electron Microscopy in Diagnostic Virology: A Practical Guide and Atlas. Can J Vet Res 52: 285.

Ducatelle R, Coussement W, Pensaert MB, Debouck P, Hoorens J (1981) In vivo morphogenesis of a new porcine enteric coronavirus. CV 777. Arch Virol 68: 35-44.

Gao Y, Kou Q, Ge X, Zhou L, Guo X, Yang H (2013) Phylogenetic analysis of porcine epidemic diarrhea virus field strains prevailing recently in China. Arch Virol 158: 711-715.

Godet M, Grosclaude J, Delmas B, Laude H (1994) Major receptor-binding and neutralization determinants are located within the same domain of the transmissible gastroenteritis virus (coronavirus) spike protein. J Virol 68: 8008-8016.

Hofmann M, Wyler R (1988) Propagation of the virus of porcine epidemic diarrhea in cell culture. J Clin Microbiol 26: 2235-2239.

Kweon CH, Lee JG, Han MG, Kang YB (1997) Rapid diagnosis of porcine epidemic diarrhea virus infection by polymerase chain reaction. J Vet Med Sci 59: 231-232.

Larsen JE, Lund O, Nielsen M (2006) Improved method for predicting linear B-cell epitopes. Immunome Res 2: 2 .

Lee HK, Yeo SG (2003) Cloning and sequence analysis of the nucleocapsid gene of porcine epidemic diarrhea virus. Virus Genes 26: 207-212.

Ogawa H, Taira O, Hirai T, Takeuchi H, Nagao A, Ishikawa Y, Tuchiya K, Nunoya T, Ueda S (2009) Multiplex PCR and multiplex RT-PCR for inclusive detection of major swine DNA and RNA viruses in pigs with multiple infections. J Virol Methods 160: 210-214.
Park SJ, Moon HJ, Luo Y, Kim HK, Kim EM, Yang JS, Song DS, Kang BK, Lee CS, Park BK (2008) Cloning and further sequence analysis of the ORF3 gene of wildand attenuated-type porcine epidemic diarrhea viruses. Virus Genes 36: 95-104.

Park SJ, Moon HJ, Yang JS, Lee CS, Song DS, Kang BK, Park BK (2007) Sequence analysis of the partial spike glycoprotein gene of porcine epidemic diarrhea viruses isolated in Korea. Virus Genes 35: 321-332.

Pensaert MB, de Bouck P (1978) A new coronavirus-like particle associated with diarrhea in swine. Arch Virol 58: 243-247.

Puranaveja S, Poolperm P, Lertwatcharasarakul P, Kesdaengsakonwut S, Boonsoongnern A, Urairong K, Kitikoon P, Choojai P, Kedkovid R, Teankum K, Thanawongnuwech $R$ (2009) Chinese-like strain of porcine epidemic diarrhea virus, Thailand. Emerg Infect Dis 15: 1112-1115.

Song D, Park B (2012) Porcine epidemic diarrhea virus: a comprehensive review of molecular epidemiology, diagnosis, and vaccines. Virus Genes 44: 167-175.

Sun D, Feng L, Shi H, Chen J, Cui X, Chen H, Liu S, Tong Y, Wang Y, Tong G (2008) Identification of two novel $B$ cell epitopes on porcine epidemic diarrhea virus spike protein. Vet Microbiol 131: 73-81.

Sun DB, Feng L, Shi HY, Chen JF, Liu SW, Chen HY, Wang YF (2007) Spike protein region (aa 636789) of porcine epidemic diarrhea virus is essential for induction of neutralizing antibodies. Acta Virol 51: 149-156.

Sun RQ, Cai RJ, Chen YQ, Liang PS, Chen DK, Song CX (2012) Outbreak of Porcine Epidemic Diarrhea in Suckling Piglets, China. Emerg Infect Dis 18: 161-163.

Tamura K, Peterson D, Peterson N, Stecher G, Nei M, Kumar S (2011) MEGA5: molecular evolutionary genetics analysis using maximum likelihood, evolutionary distance, and maximum parsimony methods. Mol Biol Evol 28: 2731-2739.

Wang L, Byrum B, Zhang Y (2014) New variant of porcine epidemic diarrhea virus, United States. Emerg Infect Dis 20: 917-919. 